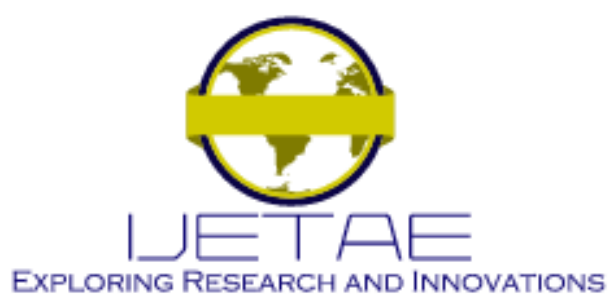

International Journal of Emerging Technology and Advanced Engineering

Website: www.ijetae.com (E-ISSN 2250-2459, Scopus Indexed, ISO 9001:2008 Certified Journal, Volume 11, Issue 07, July 2021)

Manuscript Received: 25 June 2021, Received in Revised form: 15 July 2021, Accepted: 20 July 2021

DOI: 10.46338/ijetae0721_01

\title{
Indonesian License Plate Detection and Identification using Deep Learning
}

\author{
Nico Ricky Adytia ${ }^{1}$, Gede Putra Kusuma ${ }^{2}$ \\ Computer Science Department, BINUS Graduate Program - Master of Computer Science, Bina Nusantara University, Jakarta, \\ Indonesia, 11480
}

\begin{abstract}
License plate is the unique identity of the vehicle, which serves as proof of the legitimacy of the operation of the vehicle in the form of a plate or other material with certain specifications issued by the police and contains the area code, registration number and validity period and installed on the vehicle. License plates are often used in automated parking systems and vehicle identification in case of traffic violations. So, it is necessary to build a system for detection and identification of license plates. The proposed license plate detection and identification system is divided into three main processes, namely license plate detection, character segmentation, and character recognition. The detection process uses transfer learning techniques using Faster R-CNN Inception V2. The segmentation process uses traditional computer vision with morphological operations and contours extraction. Then the character recognition process uses the MobileNet V2 transfer learning technique as an architecture for character classification. The recognition accuracy compared between MobileNet V2 and TesseractOCR shows that MobileNet V2 is superior with an accuracy rate of $96 \%$, while Tesseract-OCR has a poor accuracy of $59 \%$.
\end{abstract}

Keywords - Deep Learning, Convolutional Neural Network, License Plate Detection, Character Segmentation, Character Recognition.

\section{INTRODUCTION}

Vehicle plate detection and recognition is an important technology in traffic systems. This technology utilizes image processing to identify a vehicle from a license plate image. The variety of conditions in the field which is not necessarily ideal in the identification process poses a challenge in accuracy such as weather conditions, license plate quality and differences in intensity due to lights or the environment which can cause problems during license plate recognition. Each country has a license plate standard that is different from one another. For Indonesia, license plates have several types of background color and text color, besides that there is also information on the validity period of the license plate itself.
On the edge of the license plate there is an edge that is the same color as the text of the license plate. The following are the types of license plates in Indonesia [1]:

- Black background color with white text is personal or rental vehicle.

- Yellow background with Black text is for public transportation.

- The red background color with white text is for Government vehicles.

- The white or red background color with black text is for foreign diplomatic corps vehicles.

- Black background color with white text consisting of 5 digits and country code numbers printed smaller with sub-section format is intended for vehicles of foreign diplomatic corps operational staff.

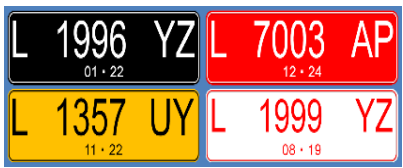

Figure 1. Various types of Indonesian vehicle license plates

Several studies on Automatic License plate Recognition (ANPR) have been carried out using various methods. The current method that is widely used for license plate detection is the method based on image processing [2], [3] and deep learning based methods [4]-[6]. Conditions in Indonesia are certainly different from other countries, such as differences in vehicle types, differences in the shape and color of license plates, and additional information in the form of the validity period of the license plates. Due to the different types of vehicles, the shape of the license plate color and the presence of a frame on the edge of the license plate and other variations, it is difficult to find a public dataset that is the same as the field conditions in Indonesia. Most publicly available datasets generally have a white background color and black text. 


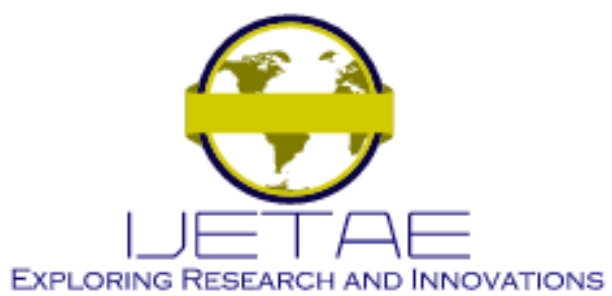

International Journal of Emerging Technology and Advanced Engineering

Website: www.ijetae.com (E-ISSN 2250-2459, Scopus Indexed, ISO 9001:2008 Certified Journal, Volume 11, Issue 07, July 2021)

Dataset is made in accordance with the state of license plates in Indonesia by collecting license plate images of various types of vehicles, plate colors, shooting angles, light, weather, and resolution. The license plate detection technique uses the Faster R-CNN model architecture [7], then character segmentation using image processing [8], [9], CNN [10] is performed to read segmented characters. The contribution of this research is proposing another method for improvisation to the adjustment of conditions in Indonesia when the detection process uses a dataset of Indonesian license plate vehicles and performs morphological operations and filter contour [11] when segmenting the required characters.

\section{RELATED WORKS}

There are many system developments that follow Computer Vision's example, such as object detection [12], [13], image recognition [14], [15], as well as certain pattern recognition. The development of this system becomes a functionality that can facilitate work in various fields. The development of deep learning [16] this is very appropriate and effective to be used to solve these problems. This cannot be separated from the research in this field. Previous research on deep learning using convolutional neural networks has been carried out by researchers on various kinds of objects.

\section{A. Transfer Learning}

Transfer learning (TL) [17], [18], is a learning enhancement technique in dealing with new tasks through the transfer of knowledge from related tasks that have been previously studied. The use of transfer learning itself is currently becoming very popular to be combined with neural networks, especially in the field of computer vision. this is because the neural network requires large data and large computing capabilities, so that combined with transfer learning can ease the computational burden, cope with large data needs, and save time during learning. The quality of a transfer learning is greatly influenced by the quality of the dataset used. Although there are no clear rules regarding the best dataset topology, the use of various objects such as dynamic object scaling and translation, randomized positioning, variable lighting, and camera angle, pose estimation, distractor noise etc.

\section{B. Automatic License Plate Recognition (ALPR)}

Image processing-based methods have previously been carried out with the proposed method which is a method consisting of two main phases, plate localization and character recognition. Plate localization is performed by combining the modified horizontal edge processing, modified vertical edge processing, region of interest extraction and short object elimination. The character recognition is done by a modified template matching algorithm with some improvements in noise removal. Another method proposed is using image processing, which consists of six main processes in this system. In preprocessing, the system will enhance frame by using top hat and bottom hat transformation. It will detect and crop the plate by using integral protection. After getting the plate position, segmentation in each character then a feature extraction combined with Artificial Neural Network get the character of the vehicle registration number.

The deep learning-based method was previously carried out with the Front-View Detection stage where the detection was initially carried out to identify the front view of the vehicle then the license plate detection was carried out. The license plate that has been detected is then carried out for character identification. Another method proposed is the initial process of directly detecting the license plate, after the position of the license plate is detected, the filter process is carried out so that the detected license plate is truly precise. The filtered license plates are then identified as the final stage. Another method uses the transfer learning object detection API using the Faster R-CNN Inception $\mathrm{V} 2$ architecture, then the recognition process uses Tesseract OCR [19].

\section{PROPOSED METHOD}

This section describes in detail the proposed approach to detection and identification of license plates in Indonesia, divided into three main stages, namely license plate detection, character segmentation [20], [21], and character identification [22], [23]. License plate detection is done to find the location of the license plate on an image. Character segmentation aims to separate and filter characters on the license plate that have been detected. Characters that have been separated and filtered are then identified so that the letters and numbers listed on the license plate are known. 


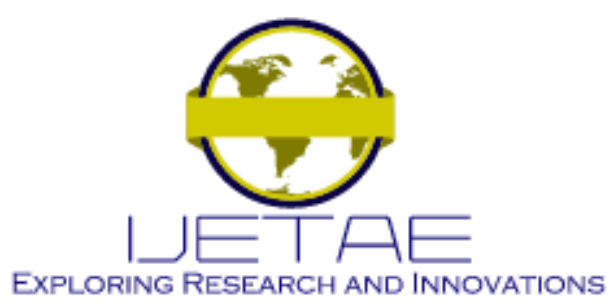

International Journal of Emerging Technology and Advanced Engineering

Website: www.ijetae.com (E-ISSN 2250-2459, Scopus Indexed, ISO 9001:2008 Certified Journal, Volume 11, Issue 07, July 2021)

\section{A. Licence Plate Detection}

Direct object detection is carried out to find the license plate to be identified. This is intended to shorten the detection process by not detecting the car first, which will certainly increase the process and execution time. The detection process uses the TensorFlow [24] object detection API model architecture Faster Region-based Convolutional Neural Network (Faster RCNN) Inception V2. The dataset that has been trained with transfer learning produces a model which is then used to detect license plates. The flow of the object detection process is described in Figure 2.

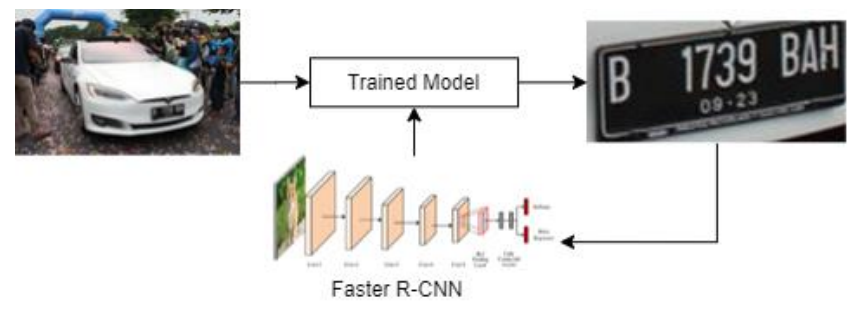

Figure 2. Process Flow of License Plates Detection

\section{B. Character Segmentation}

The license plate that has been detected is then processed as a new image which will then be carried out by the character segmentation process. Before performing the segmentation process, a filter process using morphological operations is carried out. The morphological operations used initially change the license plates to greyscale, blur, binary, and dilation. The purpose of this morphology is to sharpen the difference between writing and the background. Morphological operations produce a black background and white text. Due to the license plates in Indonesia, there is additional information on the validity period of the license plates and the presence of a white frame, this must be filtered so that only the license plate information will be used for further processing. The white frame is sometimes read as characters due to the tilted position of the license plate. The filter is done by comparing the ratio of the length and width of the white character to the width of the license plate. The illustration of the flow of the character segmentation process is described in Figure 3.

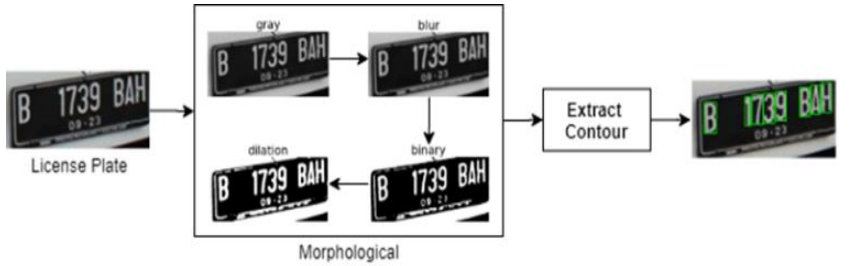

Figure 3. Process Flow of Character Segmentation

\section{Character Recognition}

Characters that have been segmented are then identified in sequence. The identification process uses the MobileNet V2 architecture whose models can be directly imported from the Keras framework [25], [26]. The advantage of this architecture is that there is very little computing power to run transfer learning. It is very suitable for use in image classification of license plate characters because of its less complex complexity. The illustration of the recognition process flow is depicted in Figure 4.

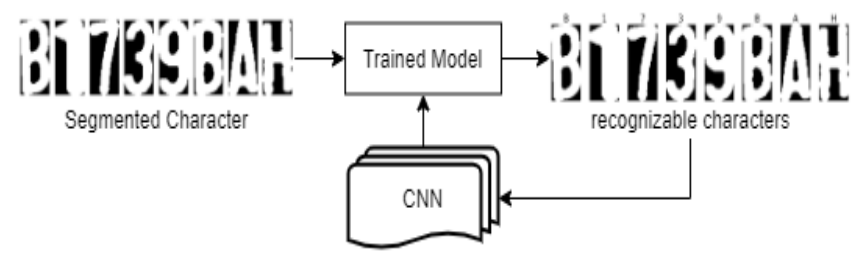

Figure 4. Process Flow of Character Identification

\section{RESUlTS AND ANALYsis}

The license plate recognition system uses two different scenarios for the test process. The first scenario for license plate recognition uses the MobileNet V2 Feature Extractor, which previously performed character segmentation. The second scenario is number plate recognition using Tesseract-OCR 4.1.1. For the number plate detection process both use the Faster R-CNN Inception V2 architecture. The training process uses Google Colaboratory Pro [27] as many as 200,000 steps integrated with Google Drive. The results of the number plate object detection training process show an accuracy of $99 \%$. The following is the display result of the TensorBoard shown in figure 5 . 


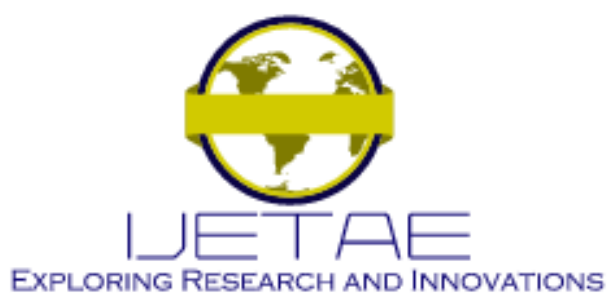

International Journal of Emerging Technology and Advanced Engineering Website: www.ijetae.com (E-ISSN 2250-2459, Scopus Indexed, ISO 9001:2008 Certified Journal, Volume 11, Issue 07, July 2021)

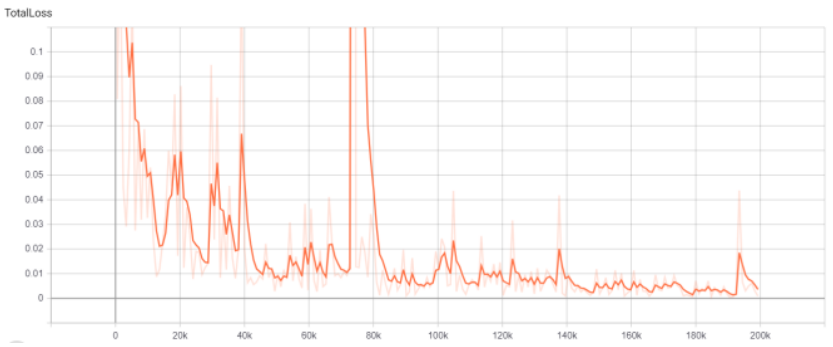

Figure 5. Total Loss of License Plate Detection

In the character identification section, the training process uses transfer learning from the MobileNet V2 architecture with pre-trained weight. The model results show an accuracy of $95 \%$ after 5 epochs and when the training process is complete, the accuracy is $99 \%$ as shown in the graph of Figure 6.
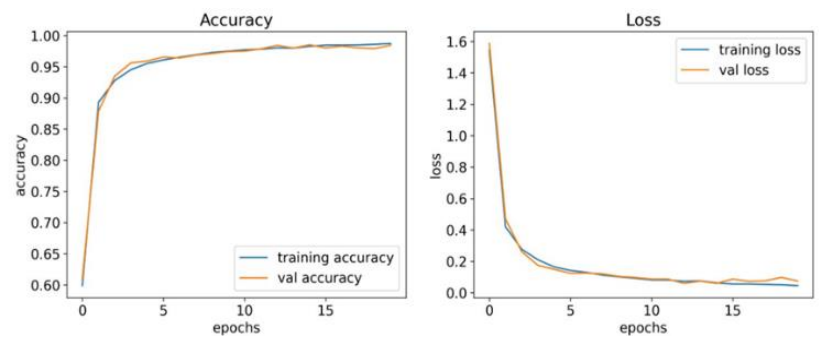

Figure 6. Training and Evaluation Accuracy
The technique of measuring the accuracy of number plate character recognition uses a comparison between the expected characters and the predicted characters. Test uses images of various types of vehicles and color license plate.

$$
\text { CR Similarity }=\frac{\text { Total Identfied Characters }}{\text { Total Factual Characters }} \times 100
$$

The following formula is to calculate the average of character identification accuracy between MobileNet V2 and Tesseract OCR 4.1.1.

$$
\bar{x}=\frac{1}{n} \sum_{i=1}^{n} \frac{C I_{i}}{C T_{i}} * 100 \%
$$

The following are the details of the calculation results of each accuracy of the number plate and then determine the average of the entire sample tested. The test results are shown in the Table 1.

Table 1.

\begin{tabular}{|c|c|c|c|c|c|c|}
\hline \multirow[b]{2}{*}{ No. } & \multirow[b]{2}{*}{ License Plate } & \multirow{2}{*}{$\begin{array}{c}\text { Factual Plate } \\
\text { Number }\end{array}$} & \multirow{2}{*}{$\begin{array}{c}\text { Proposed } \\
\text { Method Result }\end{array}$} & \multirow{2}{*}{$\begin{array}{c}\text { Tesseract OCR } \\
\text { Result }\end{array}$} & \multicolumn{2}{|c|}{ \% Similarity } \\
\hline & & & & & $\begin{array}{c}\text { Proposed } \\
\text { Method }\end{array}$ & $\begin{array}{c}\text { Tesseract } \\
\text { OCR }\end{array}$ \\
\hline 1 & D 1511 & D1511V & D1511V & none & $100 \%$ & $0 \%$ \\
\hline 2 & B 234 KIL & B234KIL & B234KIL & B234KIL & $100 \%$ & $100 \%$ \\
\hline 3 & 27665 व & Z7665TA & Z7665TA & 77669THI & $100 \%$ & $57 \%$ \\
\hline 4 & 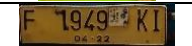 & F1949KI & F1949KI & none & $100 \%$ & $0 \%$ \\
\hline 5 & $P \cdot 841^{\prime} X X$ & P841XX & P841XX & P841XX & $100 \%$ & $100 \%$ \\
\hline 6 & E 1488 IU & L1488IU & L14881U & L1488IU & $86 \%$ & $100 \%$ \\
\hline 7 & $\begin{array}{|lll|}\mathrm{B} & 1759 & \mathrm{POH}\end{array}$ & B1759POH & B1759POH & B1759POH & $100 \%$ & $100 \%$ \\
\hline 8 & A 669 & A669U & A669U & none & $100 \%$ & $0 \%$ \\
\hline 9 & \begin{tabular}{|lll}
$B G$ & 1348 & UI \\
\end{tabular} & BG1348UI & BG1348U1 & BG1328U1 & $87 \%$ & $87 \%$ \\
\hline 10 & B $1228 \mathrm{CMU}$ & B1228CMU & B1228CMU & B1228CMU & $100 \%$ & $100 \%$ \\
\hline 11 & B $2850 \times Z 4$ & B2850XZL & B2850XZL & none & $100 \%$ & $0 \%$ \\
\hline 12 & T $1228 \cdot \mathrm{HL}$ & T1228HL & T1228HL & T4228HL & $100 \%$ & $85 \%$ \\
\hline 13 & B 2101 UFK & B2101UFK & B2101UFK & B2101UFK & $100 \%$ & $100 \%$ \\
\hline 14 & B 1709 TIE & B1709TIE & B7709TIE & B09TIE & $87 \%$ & $75 \%$ \\
\hline
\end{tabular}

Character Recognition Test Results 


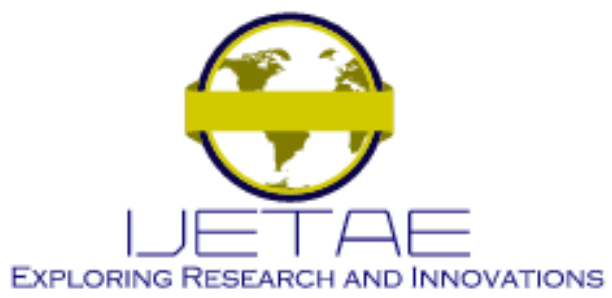

International Journal of Emerging Technology and Advanced Engineering

Website: www.ijetae.com (E-ISSN 2250-2459, Scopus Indexed, ISO 9001:2008 Certified Journal, Volume 11, Issue 07, July 2021)

\begin{tabular}{|c|c|c|c|c|c|c|}
\hline 15 & PA $1391 \mathrm{JB}$ & PA1391JB & PA1391JB & 0822 & $100 \%$ & $0 \%$ \\
\hline 16 & N 348 VR & N348VR & N348VR & none & $100 \%$ & $0 \%$ \\
\hline 17 & B 152 , RFS & B152RFS & B152RFS & B152RFS & $100 \%$ & $100 \%$ \\
\hline 18 & B 1739 BAH & B1739BAH & B1739BAH & none & $100 \%$ & $0 \%$ \\
\hline 19 & $\begin{array}{lll}\text { B } & 17 & \text { ANR }\end{array}$ & B17ANR & B17AW & B & $67 \%$ & $17 \%$ \\
\hline 20 & $\begin{array}{llll}B & 1307 & \text { UJU } \\
0.123\end{array}$ & B1307UJQ & B1307UJQ & B1307UJQ & $100 \%$ & $100 \%$ \\
\hline 21 & L 1429 ES & B1429ES & B1429ES & Ir1429ES & $100 \%$ & $86 \%$ \\
\hline 22 & BB 1741 FP & BB1741FP & BB1741FP & BB1741FP & $100 \%$ & $100 \%$ \\
\hline 23 & B 17 PSD & B17PSD & B17PSD & B17PSD & $100 \%$ & $100 \%$ \\
\hline 24 & \begin{tabular}{|llll}
$B G$ & 1 & $S T$ \\
\end{tabular} & BG1ST & BG1ST & GAST & $100 \%$ & $50 \%$ \\
\hline 25 & B .1286 FES & B1286FES & B1286FES & B1286FES & $100 \%$ & $100 \%$ \\
\hline 26 & $\sqrt{1888 \quad B}$ & N888B & N888B & none & $100 \%$ & $0 \%$ \\
\hline 27 & B 1308 RFO & B1308RFO & B1308RFO & B1308RFO & $100 \%$ & $100 \%$ \\
\hline 28 & $\begin{array}{lll} & I & 0 K 1 \\
\end{array}$ & B1DKI & B1DKI & none & $100 \%$ & $0 \%$ \\
\hline 29 & $\begin{array}{|lll|}\text { B } & 1478 & \text { TTD }\end{array}$ & B1478TTD & B1478TTD & 81478TTD & $100 \%$ & $87 \%$ \\
\hline 30 & 10040 & B1664OC & BH664QC & B1664QC & $87 \%$ & $100 \%$ \\
\hline 31 & BG 1242 IE & BG1242IE & BG12421E & eG1242IE & $87 \%$ & $87 \%$ \\
\hline 32 & $1965 \mathrm{E}$ & D1965EB & D1965 & none & $71 \%$ & $0 \%$ \\
\hline 33 & $\mathrm{KT}-1649 \mathrm{DL}$ & KT1649DL & KT1649DL & KT1649OL & $100 \%$ & $87 \%$ \\
\hline 34 & BD 7224 AN & BD7224CV & BD7224 & none & $75 \%$ & $0 \%$ \\
\hline 35 & B 2248 STW & B2248SYW & B2248SYW & ip7208StoOee & $100 \%$ & $25 \%$ \\
\hline 36 & DK $\quad \overline{46}$ & DK46S & DK46S & none & $100 \%$ & $0 \%$ \\
\hline 37 & BE $2298 \mathrm{JI}$ & BE2298JI & BE2298JI & BE2298JI & $100 \%$ & $100 \%$ \\
\hline 38 & L $9469 \times 1$ & L1469NH & L1469NH & L1469NH & $100 \%$ & $100 \%$ \\
\hline 39 & $P A 1345 R Z$ & PA1345RZ & PA1345PZ & none & $87 \%$ & $0 \%$ \\
\hline 40 & $.1025 . \mathrm{AB}$ & L1025HB & L1025HB & L1025HB & $100 \%$ & $100 \%$ \\
\hline 41 & BK 8672 C & BK8672CV & BK8672CV & none & $100 \%$ & $0 \%$ \\
\hline 42 & {$\left[\begin{array}{lll}1184 & C C \\
\end{array}\right.$} & L1184CC & L1184CC & L11ECC & $100 \%$ & $71 \%$ \\
\hline 43 & $B, 2860 \mathrm{BFC}$ & B2860BFC & B2860BFC & $82860 \mathrm{BFC}$ & $100 \%$ & $87 \%$ \\
\hline 44 & B 9005 HW & B9005HW & B9005HH & none & $86 \%$ & $0 \%$ \\
\hline 45 & $1798 \times N$ & L1798XN & L198N & L1798XN & $71 \%$ & $100 \%$ \\
\hline 46 & $\begin{array}{lll}B & 1418 & S G D\end{array}$ & B1418SGD & B1418S6D & none & $87 \%$ & $0 \%$ \\
\hline 47 & $\begin{array}{lll}1445 & 0 Z\end{array}$ & Z1445DZ & Z1445DZ & Z1445OZ & $100 \%$ & $86 \%$ \\
\hline 48 & B 10L1 RF & B1041RFZ & B1041RFZ & B1041RFZ & $100 \%$ & $100 \%$ \\
\hline 49 & B 1257 UJH & B1257UJH & B1257UJ & none & $87 \%$ & $0 \%$ \\
\hline 50 & DP' 1129 AW & DP1129AW & DP1129AH & OP1129AW & $87 \%$ & $87 \%$ \\
\hline 51 & L $1652 \mathrm{SH}$ & L1652SH & L1652SH & L1652SH & $100 \%$ & $100 \%$ \\
\hline 52 & B 1658 PVE & B1658PVE & B1658PVE & B1658PVE & $100 \%$ & $100 \%$ \\
\hline 53 & $\sqrt{102 \pi} \mathrm{T}$ & L162TJ & L162TJ & LEE18J & $100 \%$ & $50 \%$ \\
\hline 54 & Bil $1439 \mathrm{TP}$ & BM1439TP & BW1439TP & BY1740539TP & $87 \%$ & $75 \%$ \\
\hline
\end{tabular}




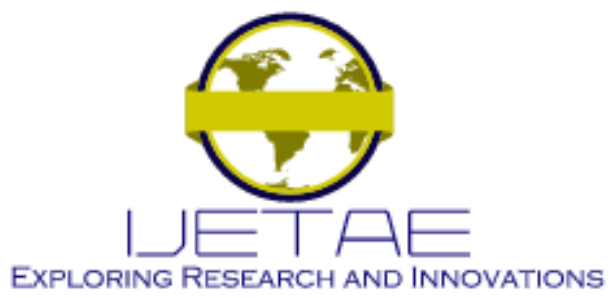

International Journal of Emerging Technology and Advanced Engineering Website: www.ijetae.com (E-ISSN 2250-2459, Scopus Indexed, ISO 9001:2008 Certified Journal, Volume 11, Issue 07, July 2021)

\begin{tabular}{|c|c|c|c|c|c|c|}
\hline 55 & $B 2805 \mathrm{UZ}$ & B2805UZ & B2805UZ & none & $100 \%$ & $0 \%$ \\
\hline 56 & 1699 SEC & B1699SEC & B1699SEC & B1699SEC & $100 \%$ & $100 \%$ \\
\hline 57 & $1582 \mathrm{BOH}$ & B1582BOH & B1582BOH & B1582BOH & $100 \%$ & $100 \%$ \\
\hline 58 & B $1980 \mathrm{KY}$ & B1980YY & B1980YY & none & $100 \%$ & $0 \%$ \\
\hline 59 & AD $9021 \mathrm{AL}$ & AD9021AL & AD9021AL & AD9027AL & $100 \%$ & $87 \%$ \\
\hline \multicolumn{5}{|c|}{ Average Similarity } & $96 \%$ & $59 \%$ \\
\hline
\end{tabular}

\section{CONCLUSION}

Research on the detection and identification of Indonesian number plates has been carried out using deep learning. The dataset used for detection of Indonesian vehicle number plates is 295 files that have been annotated with number plate data from various types of vehicles, weather conditions, light conditions, license plate conditions, vehicle position, resolution, and image quality. While the dataset used for character identification is 34,575 images which are divided into 36 characters of various shapes, orientations, and thicknesses.

The number plate identification and detection process use three main stages, namely License Plate Detection (LPD), Character Segmentation (CS), Character Recognition (CR). The LPD process uses object detection technology by means of transfer learning using the Faster $\mathrm{R}-\mathrm{CNN}$ Inception V2 architecture which has a balance between accuracy and processing time. The CR process uses traditional computer vision, namely by performing morphological operations and extract contours to filter the expected characters. Then the CR Process tries to compare between Tesseract OCR 4.1.1 and image classification technology by means of transfer learning using MobileNet V2.

The result of the comparison between MobileNet and Tesseract OCR in the character identification process proves that MobileNet V2 is far superior. Tesseract OCR has a weakness if the position of the license plate is tilted, or the camera position is not straight resulting in inaccurate results.

\section{REFERENCES}

[1] Chief of the Indonesian National Police, Peraturan Kepala Kepolisian Negara Republik Indonesia (Perkapolri) Nomor 5 Tahun 2012 pasal 39 ayat 3. Indonesia, 2012, p. undefined.

[2] Y. Hariyani and I. Wijayanto, "Indonesian Vehicle Plate Recognition and Identification Based on Digital Image Processing and Artificial Neural Network," Nov. 2012. doi: 10.13140/2.1.1917.6481.
[3] P. Hidayatullah, F. Feirizal, H. Permana, Q. Mauluddiah, and A. Dwitama, "License Plate Detection and Recognition for Indonesian Cars," International Journal on Electrical Engineering and Informatics, vol. 8, pp. 331-346, Nov. 2016, doi: 10.15676/ijeei.2016.8.2.7.

[4] J. Singh and B. Bhushan, "Real Time Indian License Plate Detection using Deep Neural Networks and Optical Character Recognition using LSTM Tesseract," Nov. 2019, pp. 347-352. doi: 10.1109/ICCCIS48478.2019.8974469.

[5] S. Montazzolli and C. Jung, "Real-Time Brazilian License Plate Detection and Recognition Using Deep Convolutional Neural Networks," Nov. 2017. doi: 10.1109/SIBGRAPI.2017.14.

[6] [6] H. Li, P. Wang, and C. Shen, "Toward End-to-End Car License Plate Detection and Recognition With Deep Neural Networks," IEEE Transactions on Intelligent Transportation Systems, vol. 20, no. 3, pp. 1126-1136, 2019, doi: 10.1109/TITS.2018.2847291.

[7] S. Ren, K. He, R. B. Girshick, and J. Sun, "Faster R-CNN: Towards Real-Time Object Detection with Region Proposal Networks," CoRR, vol. abs/1506.01497, 2015, [Online]. Available: http://arxiv.org/abs/1506.01497

[8] M. Beyeler, OpenCV with Python blueprints. Packt Publishing Ltd, 2015.

[9] L. D. L. D. Huỳnh, Y. Xu, and T. Géraud, "Morphology-based hierarchical representation with application to text segmentation in natural images," in 2016 23rd International Conference on Pattern Recognition (ICPR), 2016, pp. 4029-4034. doi: 10.1109/ICPR.2016.7900264.

[10] T. Ghosh et al., "Bangla handwritten character recognition using MobileNet V1 architecture," Bulletin of Electrical Engineering and Informatics, vol. 9, no. 6, pp. 2547-2554, 2020.

[11] M. Ren, J. Yang, and H. Sun, "Tracing boundary contours in a binary image," Image and Vision Computing, vol. 20, pp. 125-131, Nov. 2002, doi: 10.1016/S0262-8856(01)00091-9.

[12] H. Yoon, S.-H. Lee, and M. Park, "TensorFlow with user friendly Graphical Framework for object detection API," arXiv preprint arXiv:2006.06385, 2020.

[13] M. Noman, V. Stankovic, and A. Tawfik, "Object detection techniques: overview and performance comparison," in 2019 IEEE International Symposium on Signal Processing and Information Technology (ISSPIT), 2019, pp. 1-5.

[14] K. He, X. Zhang, S. Ren, and J. Sun, "Deep residual learning for image recognition," in Proceedings of the IEEE conference on computer vision and pattern recognition, 2016, pp. 770-778.

[15] B. Zoph, V. Vasudevan, J. Shlens, and Q. V Le, "Learning transferable architectures for scalable image recognition," in Proceedings of the IEEE conference on computer vision and pattern recognition, 2018, pp. 8697-8710. 


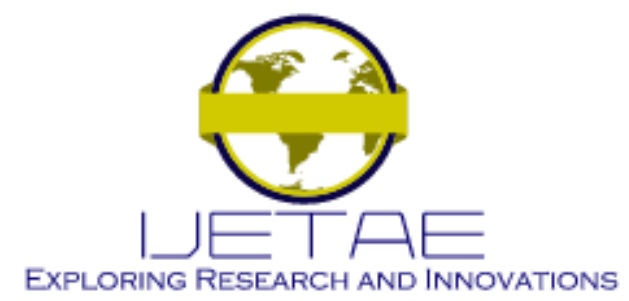

International Journal of Emerging Technology and Advanced Engineering Website: www.ijetae.com (E-ISSN 2250-2459, Scopus Indexed, ISO 9001:2008 Certified Journal, Volume 11, Issue 07, July 2021)

[16] I. Goodfellow, Y. Bengio, A. Courville, and Y. Bengio, Deep learning, vol. 1, no. 2. MIT press Cambridge, 2016.

[17] M. Hussain, J. J. Bird, and D. R. Faria, "A study on cnn transfer learning for image classification," in UK Workshop on Computational Intelligence, 2018, pp. 191-202.

[18] J. Talukdar, S. Gupta, P. Rajpura, and R. Hegde, "Transfer Learning for Object Detection using State-of-the-Art Deep Neural Networks," Nov. 2018, pp. 78-83. doi: 10.1109/SPIN.2018.8474198.

[19] N. Pawar, Z. Shaikh, P. Shinde, and Y. P. Warke, "Image to text conversion using tesseract," Image, vol. 6, no. 02, 2019.

[20] S. Stewart and B. Barrett, "Document image page segmentation and character recognition as semantic segmentation," in Proceedings of the 4th International Workshop on Historical Document Imaging and Processing, 2017, pp. 101-106.

[21] S. Kumari, L. Gupta, and P. Gupta, "Automatic License Plate Recognition Using OpenCV and Neural Network," International Journal of Computer Science Trends and Technology (IJCST), vol. 5, no. 3, pp. 114-118, 2017.
[22] A. Shalakhmetov and S. Aubakirov, "Optical character recognition with neural networks," Journal of Mathematics, Mechanics and Computer Science, vol. 100, no. 4, pp. 28-41, 2019.

[23] A. Chaudhuri, K. Mandaviya, P. Badelia, and S. K. Ghosh, "Optical character recognition systems," in Optical Character Recognition Systems for Different Languages with Soft Computing, Springer, 2017, pp. 9-41.

[24] J. v Dillon et al., "Tensorflow distributions," arXiv preprint arXiv:1711.10604, 2017.

[25] A. Gulli and S. Pal, Deep learning with Keras. Packt Publishing Ltd, 2017.

[26] A. Géron, Hands-on machine learning with Scikit-Learn, Keras, and TensorFlow: Concepts, tools, and techniques to build intelligent systems. O’Reilly Media, 2019.

[27] E. Bisong, "Google Colaboratory," in Building Machine Learning and Deep Learning Models on Google Cloud Platform, Springer, 2019, pp. 59-64. 